\title{
Current Sheet Formation in a Conical Theta Pinch Faraday Accelerator with Radio-frequency Assisted Discharge
}

\author{
IEPC-2007-165 \\ Presented at the $30^{\text {th }}$ International Electric Propulsion Conference, Florence, Italy \\ September 17-20,2007 \\ Ashley K. Hallock* and Edgar Y. Choueiri ${ }^{\dagger}$ \\ Princeton University, Princeton, NJ, 08540, USA \\ Kurt A. Polzin ${ }^{\ddagger}$ \\ NASA - Marshall Space Flight Center, Huntsville, AL, 35812, USA
}

\begin{abstract}
The inductive formation of current sheets in a conical theta pinch FARAD (Faraday Accelerator with Radio-frequency Assisted Discharge) thruster is investigated experimentally with time-integrated photography. The goal is to help in understanding the mechanisms and conditions controlling the strength and extent of the current sheet, which are two indices important for FARAD as a propulsion concept. The profiles of these two indices along the inside walls of the conical acceleration coil are assumed to be related to the profiles of the strength and extent of the luminosity pattern derived from photographs of the discharge. The variations of these profiles as a function of uniform back-fill neutral pressure (with no background magnetic field and all parameters held constant) provided the first clues on the nature and qualitative dependencies of current sheet formation. It was found that there is an optimal pressure for which both indices reach a maximum and that the rate of change in these indices with pressure differs on either side of this optimal pressure. This allowed the inference that current sheet formation follows a Townsend-like breakdown mechanism modified by the existence of a finite pressure-dependent radio-frequency-generated electron density background. The observation that the effective location of the luminosity pattern favors the exit-half of the conical coil is explained as the result of the tendency of the inductive discharge circuit to operate near its minimal self-inductance. Movement of the peak in the luminousity pattern towards the upstream side of the cone with increasing pressure is believed to result from the need of the circuit to compensate for the increase in background plasma resistivity due to increasing pressure.
\end{abstract}

\section{Nomenclature}

$$
\begin{array}{ll}
d & =\text { distance from the cone inlet } \\
n_{e} & =\text { electron number density } \\
n_{n} & =\text { neutral number density } \\
P^{*} & =\text { critical pressure } \\
\delta & =\text { half cone angle of inductive coil } \\
\nu_{e-n} & =\text { electron-neutral collision frequency }
\end{array}
$$

\footnotetext{
${ }^{*}$ Graduate Student, Mechanical and Aerospace Department, ahallock@princeton.edu.

${ }^{\dagger}$ Chief Scientist EPPDyL, choncirioprinceton.edu.

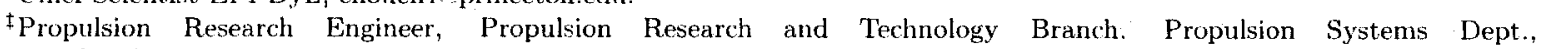
kurt.a.polzin@nasa.gov.
} 


\section{Introduction}

Electrode-less spacecraft propulsion thrusters are attractive devices due to their avoidance of lifetimelimiting electrode erosion and their ability to use electrode-incompatible propellants (such as $\mathrm{CO}_{2}$ and $\mathrm{H}_{2} \mathrm{O}$ ). Pulsed inductive thrusters ${ }^{1}$ are one type of electrode-less plasma propulsion devices. Their operation consists of a high frequency current pulse through an inductive coil that induces a time-changing magnetic field and, in accordance with Faraday's Law of Induction, an electric field. If propellant is within a certain distance of the coil, this electric field will break down neutral gas and the changing magnetic field will induce a sheet carrying current opposing that in the coil. The interaction of the current density in the sheet with the induced magnetic field produces a Lorentz body force density that will act to expel the current sheet away from the coil, entraining propellant and producing thrust.

Because the coupling between the propellant and the inductive coil affects the efficiency of the thruster, it benefits the design to provide propellant to the inductor that has already been ionized through some external means. ${ }^{2}$ A pulsed inductive propulsion device, called the Faraday Accelerator with Radio-frequency Assisted Discharge (FARAD), was first introduced by Choueiri and Polzin ${ }^{3}$ who carried out proof-of-concept experiments, derived design criteria, ${ }^{4}$ and described some potential advantages over a previous concept, the Pulsed Inductive Thruster (PIT). ${ }^{1,5,6}$ Among these potential advantages is FARAD's ability to form current sheets at much lower capacitor voltages and energies than PIT $(1.5 \mathrm{kV}, 44 \mathrm{~J}$ versus $30 \mathrm{kV}, 4 \mathrm{~kJ})$ through the use of a RF-generated preionization. This advantage incurs the added complexity of incorporating an RF plasma source and applied magnetic field, which are used to produce and guide plasma with a high-enough density for current sheet formation onto the face of a flat inductive coil. A particular problem is the difficulty of designing a non-cusped magnetic field that can effectively deliver a significant portion of the pre-ionized propellant to the coil.

The Conical Theta Pinch FARAD (CTP-FARAD) is a permutation of the original FARAD concept with the single difference that a conical inductive coil is used instead of a flat coil. Figure 1 shows, in the left panel, a schematic of the original FARAD design with the flat acceleration inductive coil. In the present work, the flat coil is is replaced by the conical coil shown in the right panel of the same figure. This geometry allows for a non-cusp applied magnetic field that more closely follows the coil's face and that should be more effective at guiding the pre-ionized propellant to the coil. Because of this conformity to the natural path of plasma diffusion, current sheets are possible in CTP-FARAD even with no applied magnetic field. Moreover, the CTP-FARAD cone is expected to produce an additional thrust component derived from electromagnetic pinching of the plasma.
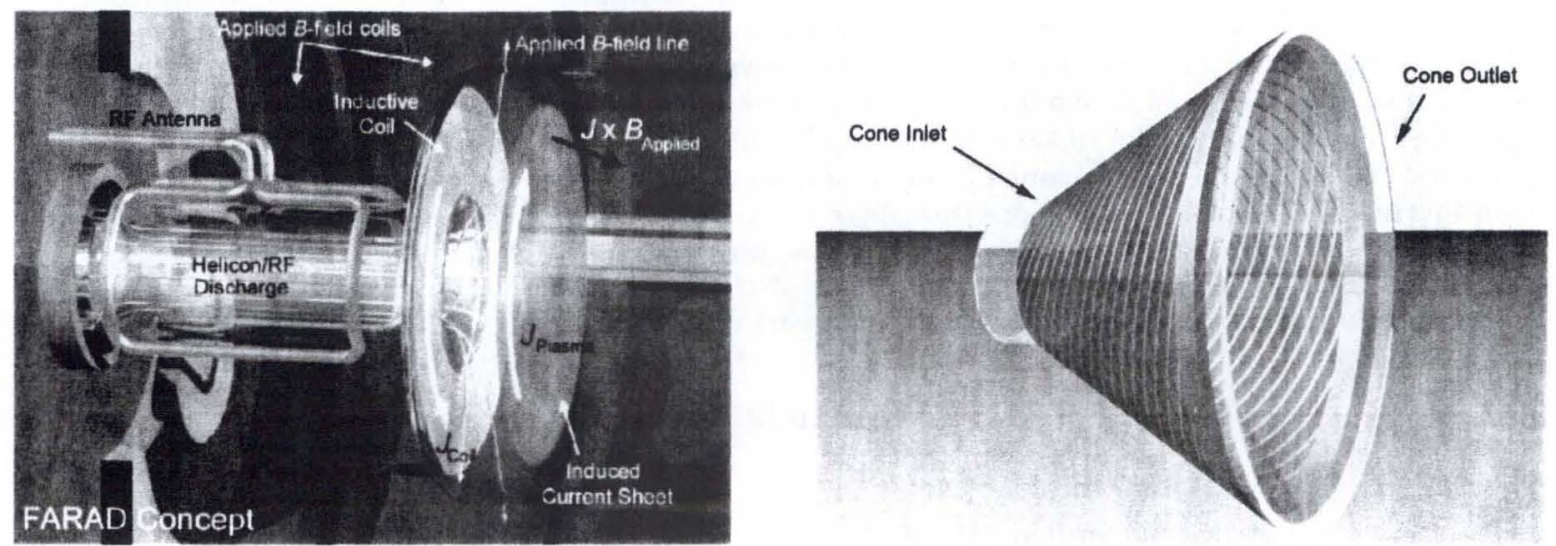

Figure 1. Left: Schematic of original FARAD set-up with flat Inductive coil. Right: Schematic of replacement conical coil.

The propulsive efficacy of the current sheet depends on a number of factors (e.g., sheet strength, extent, decoupling distance from the coil, propellant leakage, pinching, stability, etc.). Only two of these, namely strength and extent, are the focus of the present study. Specifically, the thruster should ideally induce a strong (high current-density) current sheet that uniformly extends over the entire coil surface in order to maximize the amount of work done on the plasma. An understanding of the mechanisms and conditions controlling the intensity and extent of the current sheet would be useful in providing guidance for future 
designs and in choosing operating conditions that most enhance these two sheet performance indicies with respect to propulsive efficiency.

The goal of the study reported here is to provide the first chues on the underlying mechanisms and conditions behind the formation, intensity and extent of current sheets in ('TP-FARAD. Ultimately, for a given physical configuration, we need to know how to maximize the thruster's performance as a function of the controllable operating parameters (nass How rate, propellant type, RF power, and applied magnetic ficld topology and strength). In the present study we start with our attempts at elucidating the underlying physical mechanisms by focusing on the most basic dependencies of sheet formation, namely the role of background density. While in a real thruster the background density, for a given configuration and $\mathrm{RF}$ power, is controlled by the mass flow rate through the thruster; in this basic study we chose to control the background density by varying the uniform back-fill gas pressure, while holding all controllable parameters fixed and applying no magnetic field. 'This removes the complications of the flow and expansion the preionized gas from the picture and allows for a clearer isolation of the basic mechanisms controlling current sheet formation, strength and extent.

Furthermore, before carrying out detailed studies using Langmuir probes, B-dot probes, and more analytical optical diagnostics, we limited our initial investigation to a single diagnostic: time-integrated photography. This was motivated by the visually rich character of the distinguishable visible-light emission associated with the sheet.

The rest of the paper is organized into successive sections in which we describe the experiment, document the new conical coil design, describe the photographic data collection, report the observed trends and attempt to extract physical insight into some of the basic mechanisms underlying sheet formation, strength and extent.

\section{Experimental Setup}

\section{A. Vacuum Chamber}

All experiments were performed in a vacuum chamber consisting of two pyrex cylinders joined together by a fiberglass plate on which the CIP is mounted, extending $12 \mathrm{~cm}$ into the larger cylinder. 'The smaller cylinder has an inner diameter of $6 \mathrm{~cm}$ and is $37 \mathrm{~cm}$ long and the larger cylinder has an inner diameter of $20 \mathrm{~cm}$ and is $46 \mathrm{~cm}$ long. While the experiment can be operated with mass injection through the endplate of the smaller cylinder, for the present study the chamber is back-filled to various pressures from a port on the opposite end of the experiment. 'The pressure is monitored using a Granville Phillips 275 mini-convectron pressure gauge. Plasma is created in the smaller, pre-ionization chamber and diffuses through a 6 cm inmer diameter concentric hole in the fiberglass plate into the region bounded by the CTP before eventually migrating into the remainding volume of the larger cylinder. A $150 \mathrm{l} / \mathrm{s}$ turbomolecular pump backed by a roughing pump is attached to the larger cylinder at the endplate opposite the CTP inductive coil; the same endplate where gas is fed into the vacuun vessel.

\section{B. Plasma Source}

A Boswell-type saddle antenna is wrapped around the smaller cylinder, creating the pre-ionization chamber. The saddle is composed of quarter-inch copper tubing attached to a water cooling line. An ENI 13.56 MHz $1.2 \mathrm{~kW}$ power supply is coupled to the antenna through a tuner consisting of two Jennings $1000 \mathrm{pF} 3 \mathrm{kV}$ variable vacuum capacitors located immediately next to the antenna and used to minimize reflected power. A Faraday cage surrounds the entire experiment to shield from radiated RF fields.

\section{Conical Theta Pinch}

The conical theta pinch is composed of a flexible circuit board wrapped around a pyrex fumel with a wall thickness of $4 \mathrm{~mm}$ and providing structural support to the circuit board. The neck of the funnel, with a $6 \mathrm{~cm}$ inner diameter and $2 \mathrm{~cm}$ length, fits into the concentric hole in the fiberglass plate holding the funnel's axis parallel to the horizontal plane. The conical coil used for the present study has a half-angle of 30 degrees. The design of the circuit board, shown in Fig. 2, is based on the design of the flat inductive coil found in the current $\mathrm{PIT}^{7}$ and the original FARAD experiment. The circuit traces follow an Archimedes spiral with current flowing down one surface from the bus at the major radius to plated through holes at the ninor radius. This current then proceeds up the back side of the circuit board (shown as lighter-colored traces in 
Fig. 2), providing cancellation of the radial component of current with the traces from the front side. In this way, the circuit produces a purely azimuthal current that is uniform across the coil face. The traces that form this azimuthal current end 1 cm before the dowustream end of the cone, and all normalization made with respect to a particular distance from the cone inlet is made with respect to the downstream end of the cone (not the edge of the inductive coil).

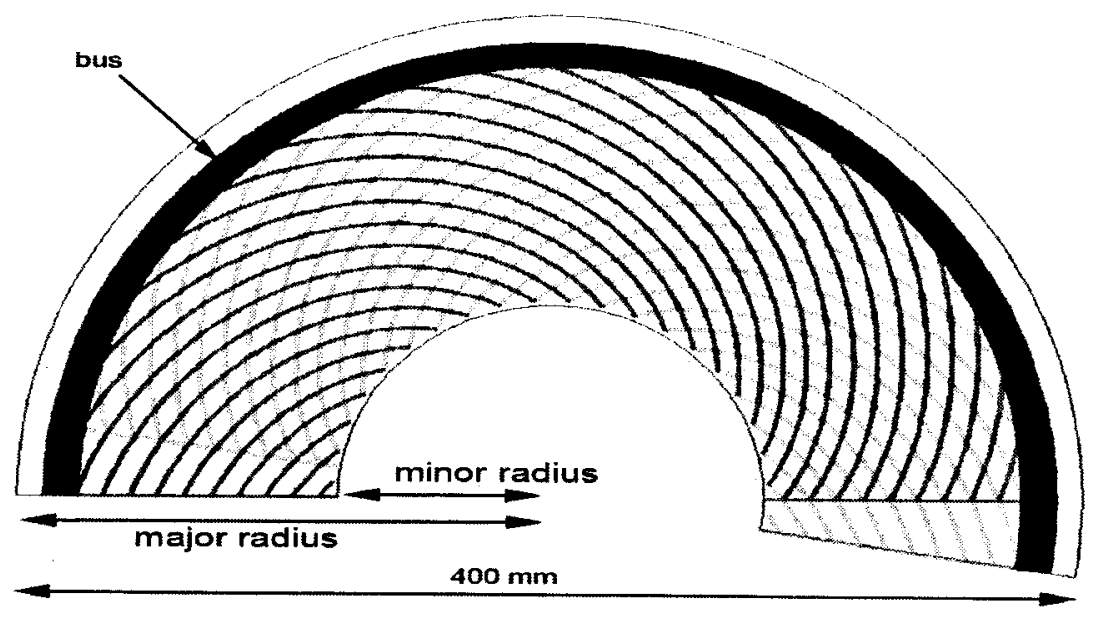

Figure 2. The CTP inductive coil shown as it is a print on a circuit board, with twenty spirals per side printed onto a .002 inch-thick Kapton substrate to maintain flexibility. Current is fed to the circuit through the bus, flows down the darker-colored traces on the front side of the circuit board, through plated through holes at the minor radius, and then up the lighter-colored traces on the back side. The ends of the board are brought together to form the conical theta pinch.

Current is fed to the coil through strip-lines, one between a $39.2 \mu \mathrm{F}$ capacitor and the vacuun vessel and another from the interior wall of the vacuum vessel fiberglass plate to the circuit-board bus (shown in Fig. 2). The circuit penetrates the vacuum vessel through metal standoffs, which connect the two striplines. The current pulse is initiated (as in the FARAD) using a "lammer" switch. When a current pulse is thus fed into the coil, a time changing radial and axial magnetic ficld and an azimuthal electric field are induced in the volume contained by the coil. If pre-ionized propellant is present within the decoupling distance of the coil, a current sheet will be induced in this propellant during the first half-cycle of the pulse at voltages an order of magnitude lower than those required for operation of the PIT. ${ }^{3}$ This current sheet will form such that, inside of the sheet, its own induced field cancels out the magnetic: field from the coil and, between the current sheet and coil, the two induced fields add. This build-up of magnetic pressure acts to accelerate the current sheet away from the coil normal to the coil surface for a uniform induced magnetic field profile.

Since the force is normal to the coil surface, the CTP inductive coil provides a radial force density on the plasma current sheet as well as an axial one. The axial component of the force density contributes to the total thrust by imparting axial directed kinetic energy to the current sheet and any entrained propellant. The radial force density component takes advantage of a portion of the pre-ionized propellant that is not involved in current sheet formation by pinching the current sheet, and can provide an additional contribution to thrust as well as reduction in the divergence of the exiting plasma. Schematics showing the idealized stages of sheet formation and pinching are shown in Fig. 3.

\section{Time-integrated Photography}

A Nikon D50 digital SLR camera with a $50 \mathrm{~mm}$ focal length lens was used to capture time-integrated images of the luminous pattern associated with the current sheet. All automatic compensation for light intensity, gamma, and color balance were defeated in the acquisition of these data. Fach picture was taken with a half second exposure time, an ISO of 100, and a preset whitebalance of uniform gray. The camera was mounted for a top view of the cone at a distance of roughly $60 \mathrm{~cm}$ from the cone exit. Due to physical constraints of the experimental setup, the camera is unable to view the cone surface at an angle where the "film plane" of the camera is parallel to the plane subtending the bottom edge of the cone. All photographs were obtained with an angle of 30 degrees between these two planes. 

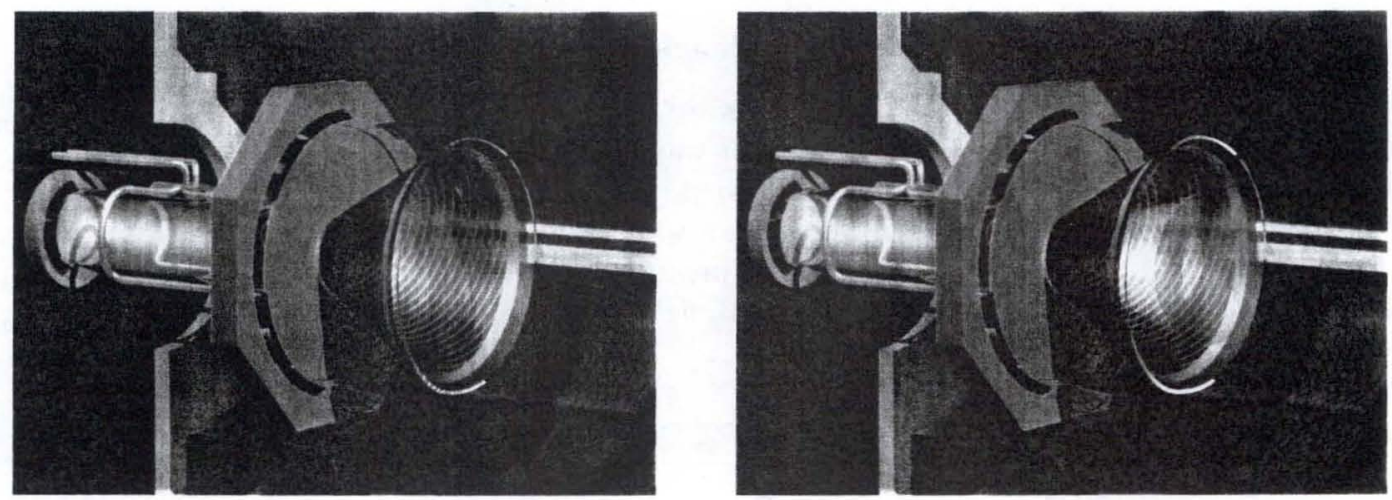

Figure 3. Idealized schematics showing current sheet formation (left panel) and pinching (right panel) in the CTP-FARAD.

The picture frame includes the lower half of the inside cone surface, allowing determination of the intensity and extent of the luminous structure from the cone's inlet to its outlet. The aperture is remotely opened, the switch is then activated sending the current pulse through the coil, and the aperture closes automatically after one half second.

\section{Experimental Results}

\section{A. Interpretation of the Images}

While the steady-state argon discharge from the RF source fills the cone with a gas radiating pink light, this distinct luminosity is greatly overpowered by an azimuthally-symmetric bright blue discharge that emanates from inside of the cone when the switch is closed. We make the assumption that the intensity, location and extent of this latter luminous structure along the wall of the cone are highly related to those of the current sheet. However, previous study of current sheets between plane electrodes ${ }^{8}$ using high-speed imaging and Bdot probes showed high correlation between the current carrying structures and their luminous signatures. In our study, the use of time-integrated photography implies a loss of some of this correlation since the luminous structure is more a representation of both the regions where the current has passed at some time during the pulse and those containing radiating gas that does not carry current. Moreover, since the current sheet presumably moves away from the cone's inner surface during the pulse, there is a parallax error associated with determining its location along the cone's surface from time-integrated photographs obtained with a camera whose film plane is not parallel to the cone's axis of symmetry.

Due to these limitations we take the measured intensity, location and extent of the luminous structure that appears during the pulse as rough representations of the corresponding features of the current sheet and limit our conclusions to only relative changes in these measured quantities.

\section{B. Image Processing}

All image processing is performed in MatLab. The images are filtered for blue light to increase the signal to noise ratio. To further increase the signal to noise ratio, a first photo that includes a current sheet is taken directly before a second photo with no current pulse (but with the background RF plasma on), and this second image is subtracted from the first to eliminate a large fraction of the background light associated with the steady-state RF discharge. This procedure is repeated five times for a given condition and the intensity values are averaged. Intensity values on a line along the cone surface are plotted with respect to distance from the cone inlet. When distance from the cone inlet is normalized, it is normalized to the cone outlet, which occurs at $13.5 \mathrm{~cm}$ from the cone inlet. The edge of the inductive coil occurs at $9.5 \mathrm{~cm}$ from the cone inlet, and corresponds to a normalized distance of .7. The resulting axial profiles are also integrated and normalized to their peak values to provide a measure of the intensity and extent of the luminous structure. 


\section{Measured Profiles and Pressure Dependencies}

The RF power source was held constant at $200 \mathrm{~W}$ forward power and $1 \%$ reflected power. No applied magnetic: filed was employed and the voltage on the capacitor was maintained at $1.25 \mathrm{kV}$ for each pulse. The argon back-fill pressure in the CTP vacuum vessel was varied from $4 \mathrm{mT}$ up to $48 \mathrm{mT}$.

Figure 1 shows intensity profiles along the cone's wall with the inlet located at $0 \mathrm{~cm}$ and the exit at $13.5 \mathrm{~cm}$. Representative profiles from eight back-fill pressure levels spanning the range of varied pressure are shown. The small peak at the latter location is due to light reflection from the downstream edge of the cone.
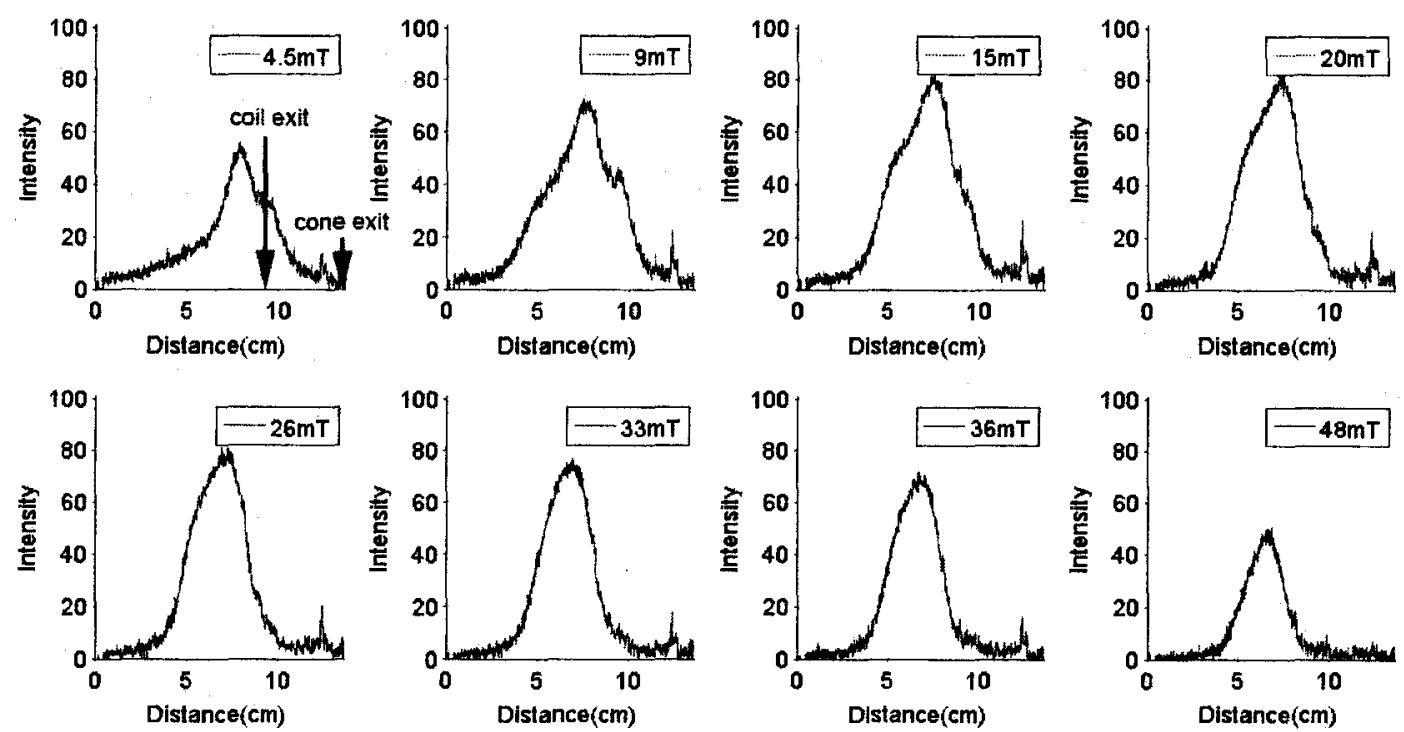

Figure 4. Profiles of intensity (in arbitrary units) versus distance from inlet along the cone's wall for eight back-fill pressure levels spanning the back-fill pressure range

Figure 5 shows the effective intensity of the current sheet plotted as a function of pressure. Effective intensity for a given pressure is calculated by integrating the curve of intensity versus position and normalizing it with respect to the maximum value for that integral attained over the pressure range.

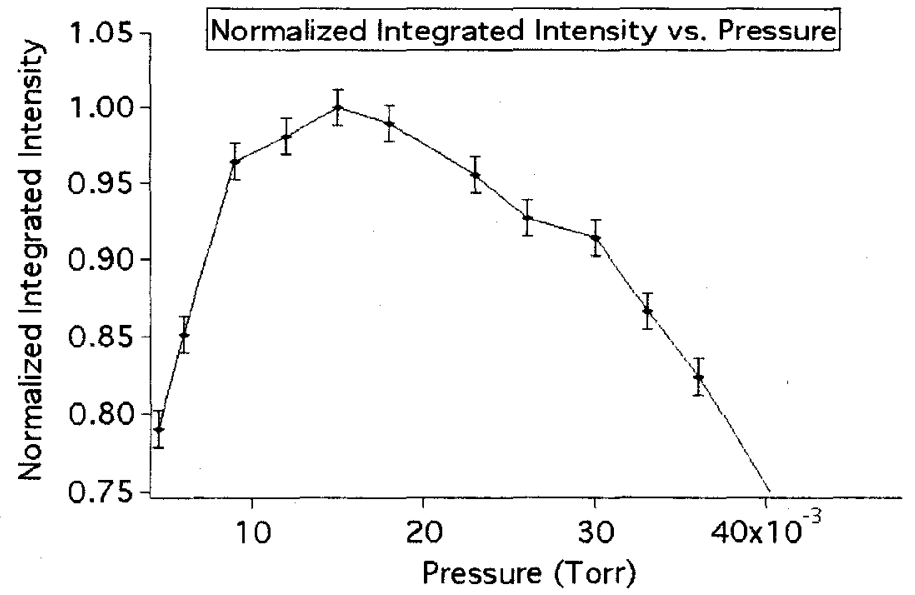

Figure 5. Effective intensity of the current sheet as a function of back-fill pressure

Figure 6 shows a plot of effective current sheet extent versus pressure. Effective current sheet extent along the wall (in the axial direction) for a given pressure is calculated as the integral of the curve of intensity 
versus position divided by the peak intensity for that pressure and normalized to the maximum current sheet extent attained over the pressure range.

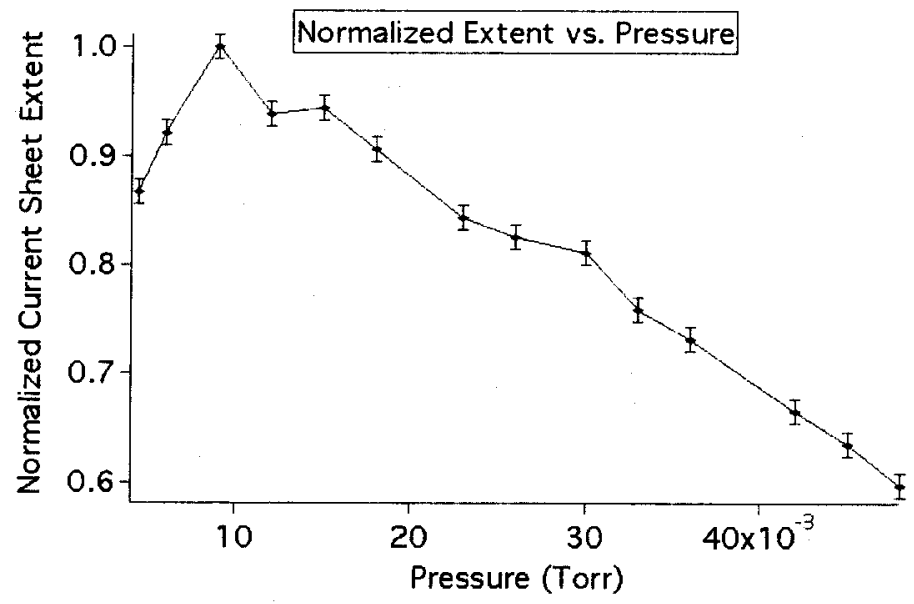

Figure 6. Effective axial extent (along the wall) of the current sheet as a function of back-fill pressure. The values of the extent are normalized by the largest value.

The effective location is determined by calculating the centroid for each curve of intensity versus distance from the cone inlet. The centroid locations are normalized by the distance between the cone inlet and exit $(13.5 \mathrm{~cm})$, with the cone inlet and beginning of the inductive coil at 0 , the inductive coil ending at .7, and the exit at 1, and plotted versus back-fill pressure in Fig. 7.

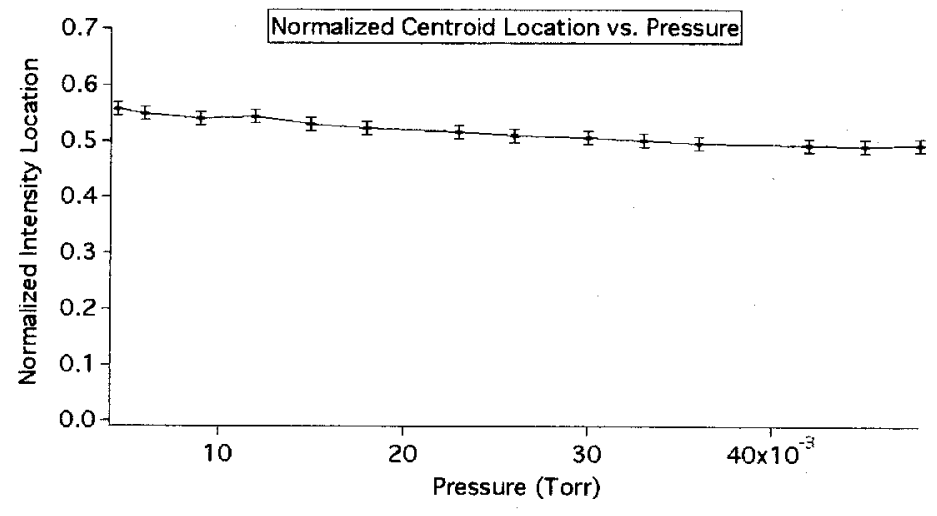

Figure 7. Effective location (along the wall) of the current sheet as a function of back-fill pressure. The locations are normalized so that the cone inlet and beginning of the inductive coil are at 0 , the end of the inductive coil is at .7 , and the cone exit is at 1 .

\section{Observations}

From the experiments and the plots of Figs. 4-7, we make the following observations:

1. Current sheets of varying intensity, extent and location were observed to form at each of the back-fill pressure levels in the investigated range ( 4 to $48 \mathrm{~m}$ Torr).

2. There is an optimal back-fill pressure, $P^{*}$ (between 10 and 15 mTorr for the particular conditions of this experiment), for which both the effective intensity and extent of the current sheet are largest (Figs. 5 and 6).

3. The rate of change in effective intensity (and extent) with pressure differs on eit her side of this optimal pressure. Specifically, the growth rate of intensity (and extent) with increasing pressure below $P^{*}$ is significantly higher than its decay rate with increasing pressure above $P^{*}$. 
4. For pressure levels below $P^{*}$, the current sheet intensity profiles are not symmetric and are relatively more extended towards the cone exit. Above $P^{*}$ they become more symmetric with respect to cone inlet and cone outlet (Fig. 1), but at all pressures the current sheet favors the downstrean end of the inductive coil.

5. For all pressure levels in the investigate range, current sheet formation occurs around the middle section of the cone but strongly favors the downstream half of the inductive coil. $\Lambda$ s can be seen in Fig 7 , current sheet formation occurs at normalized distances of 0.5 to 0.56 from the inlet, while the end of the coil occurs at .7 .

6. As the pressure rises the effective location of current sheet formation moves slightly, but definitely and monotonically, towards the inlet (Fig. 7).

We proceed by using these qualitative observations to glean some basic aspects of the mechanisms underlying current sheet formation in such a device.

\section{Discussion and Interpretation of the Observations}

The existence of an optimal pressure (Observation 2) is indicative of a Townsend-like breakdown. On the lower-pressure side of $P^{*}$, due to the relatively lower neutral density, the electrons involved in current sheet breakdown undergo fewer ionizing collisions with the background nentrals, while on the higher pressure side of that optimum they gain less energy from the electric field between collisions because their mean free path between collisions with neutrals is smaller.

Unlike in the classic Townsend breakdown, however, there is a finite steady-state backgorund electron density from the steady-state RF discharge in FARAD without which breakdown would require far larger pulse energies. Measurements of a similar $\mathrm{RF}$ argon discharge at fixed $\mathrm{RF}^{\prime}$ input power of $200 \mathrm{~W}$ were reported by Chang et al. ${ }^{9}$ and are reproduced in Fig. 8. These data show that the electron density increases with pressure over the pressure range of our experiment. (Of course this increase camnot go indefinitely at constant power as the energy input per unit mass decreases with increasing pressure.)

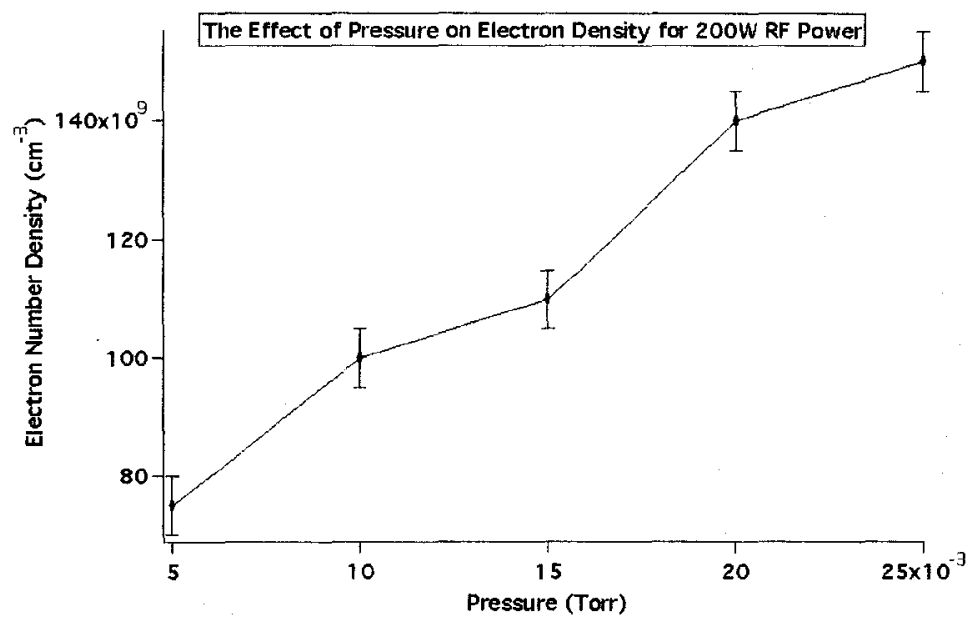

Figure 8. The effect of pressure on electron number density in a $13.56 \mathrm{MHz}$ RF inductive discharge. ${ }^{9}$

T'he reported increase of electron density with back-fill pressure implies that the decrease in ion production rate due the lower average electron energy on the high-pressure side of $P^{*}$ is, to some extent, counterbalanced by the increase in the number of background electrons. This would explain the lower rate of decrease in the current sheet's intensity and extent vs pressure at that side of the optimum relative to their increase rate on the lower-pressure side (Observation 3).

It is well know in pulsed plasma thruster research that the current sheet tends to form in such a way that the inductance presented to the circuit is minimized. ${ }^{2}$ For the conical theta pinch, this implies a current sheet forming at a location that serves to exclude as much coil-generated magnetic flux from the interior 
volume of the coil. A current sheet forming at the downstrean end of the coil circumscribes a larger area circle than a current sheet forming further upstream. This may explain why the current sheet favors the downstream location.

In addition, a current sheet forming at the downstream edge of the inductive coil will tend to affect magnetic flux lines further upstream, forcing them to stay close to the walls of the theta-pinch to maintain $\nabla \cdot \mathbf{B}=0$. A current sheet forming near the upstream end, consisting of a smaller ring current, will not have nearly the same effect on the magnetic field lines in the rest of the theta pinch's interior. Consequently, the point of lowest inductance, and the point where the sheet is predisposed to form, is at or near the downstream end of the inductive coil. This may explain why the current sheet has an asymmetric extent favoring the exit end of the coil (Observation 4) and why current shect formation (at all the investigated pressure levels) is biased (to a varying degree) towards the downstream end of the inductive coil (Observation 5 ). Because the circuit board traces end $4 \mathrm{~cm}$ before the cone outlet, current sheet formation is less dramatically biased toward the exit half of the cone as it is biased towards the downstream edge of the inductive coil.

The remaining obscrvation (Observation 6) that current sheet formation has a tendency to move upstream with increasing pressure may be explained through a consideration of the inductive circuit shown in Fig. 9.

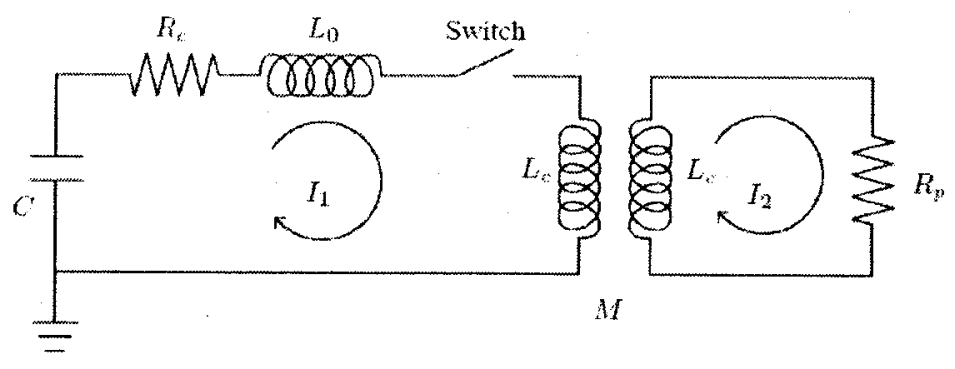

Figure 9. Schematic of the inductive circuit used to explain Observation 6

The resistivity of a weakly ionized plasma, such as the background RF-produced plasma in CTP-FARAD, is dominated by electron-neutral collisions. As the pressure increases, the frequency of these collisions increases, increasing the resistivity in the preionized plasma. During the inductive pulse, the plasma has a resistance $R_{p}$ that is equal to the resistivity integrated around the circumference of the coil. This value increases for a given axial location as a function of pressure and for a given pressure it decreases as we move axially towards the inlet. From a circuit point-of-view, we recognize that the voltage drop across the transformer must be equal to the voltage drop owing to the plasma resistance. This would require the current sheet to initially form further upstream in the theta-pinch coil as the pressure and $R_{p}$ increase at a given axial location. Consequently, in a pulsed inductive discharge the sheet may be forced to form at a location other than the point of minimum overal circuit inductance.

\section{Summary and Concluding Remarks}

A photographic study of the effects of back-fill pressure on the intensity, extent and location of current sheets in a conical theta pinch FARAD thruster provide some basic insights into the mechanisms regulating current sheet formation. The existence of an optimal pressure for maximum sheet intensity and strength and the difference in the rates of change in these quantities with changing pressure on cither side of this optimum were explained as the behavior of a Townsend-like breakdown modulated by a background electron density that increases with pressure. The effective axial location (along the wall) around which the sheet tends to form, and its dependence on pressure, are thought to be related to a dynamic balance between the resistive elements (depending on plasma resistivity and, thus, pressure) and inductive elements (depending on the axial location inside the conical coil) of an equivalent inductive circuit.

Future work will aim at verifying and consolidating these insights with more analytical diagnostics and models, with the ultinate goal of deriving prescriptions for optimizing current sheet formation and evolution as a function of cont rollable operating parameters, including the mass flow rate throigh the device, applied magnetic field and RF power. 


\section{Acknowledgments}

This research project is carried out under a contract from the the Air Force Office of Scientific Research. We also acknowledge support from the Plasma Science and Technology Program from the Princeton Plasma Physics Laboratory. We thank Mr. Robert Sorenson for his valuable technical support and for the 3-D illustrations of the CTP-FARAD concept.

\section{References}

${ }^{1}$ R.H. Lovberg C.L.Dailey. Large dianeter inductive plasma thrusters. Number AIAA 79-2093, oct 1979.

${ }^{2}$ R. G. Jahn. Physics of Electric Propulsion. McGraw-Hill Book Company, 1968.

${ }^{3} \mathrm{E}$. Y. Choueiri and K. A. Polzin. Faraday acceleration with radio-frequency assisted discharge. Journal of Propulsion and Power, 22(3):611-619, May-June 2006.

${ }^{4} \mathrm{~K}$. A. Polzin and E. Y. Choueiri. Performance optimization criteria for pulsed inductive plasma acceleration. IEEE Transactions on Plasma Science, 34(3):945 953, 2006.

${ }^{5}$ C.L. Dailey and R.H. Lovberg. Current sheet structure in an inductive-impulsive plasma accelerator. AIAA Journal, 10(2):125-192, Feb. 1972.

${ }^{6}$ R.H.Lovberg C.L.Dailey. Pit mark v design. Number AIAA 91-3571, 1991.

${ }^{7}$ R. H. Lovberg C. L. Dailey. The PIT MkV pulsed inductive thruster. 'Iechnical report, Lewis Research Center, 1993.

${ }^{8}$ T.E. Markusic and J. W. Berkery E. Y. Choueiri. Visualization of current sheet evolution in a pulsed plasma accelerator. IEEE Transactions of Plasma Science, 33(2):528-529, April 2005.

${ }^{9} \mathrm{C}$. Chang, K. Leou, and C. Lin. Real-time control of ion density and ion energy in chlorine inductively coupled plasma etch processing. Journal of vacuum Science and Technology, 21(4):1183 1187, July 2003. 